\title{
The Rate of Obesity in Psychogenic Non-Epileptic Seizures: A Retrospective Study
}

\author{
Abuhuziefa Abubakr ${ }^{\mathrm{a}, \mathrm{c}}$, Ilse Wambacq ${ }^{\mathrm{b}}$
}

\begin{abstract}
Background: Obesity is common in US population at a rate of $60 \%$. Similarly, the rate of overweight/obesity in epilepsy patients was significantly higher; however, there were no reports in patients with psychogenic non-epileptic seizures (PNESs).
\end{abstract}

Methods: We retrospectively reviewed all the records of patients admitted to the epilepsy monitoring unit with the diagnosis of PNES. Body mass index (BMI) was calculated and compared to the reported rate of obesity in epilepsy patients (55.2-72\%).

Results: The rate of overweight/obese in our cohort with PNES is $69.3 \%$, similar to what has been reported in the epilepsy population. We did not find any correlation between frequency of seizures, duration of symptom onset or antiepileptic drugs (AEDs) with positive effect on weight and the current rate of obesity in PNES patients.

Conclusion: We concluded that overweight/obesity in PNES is similar to the reported rate of the epilepsy patients and may reflect the overall trend of obesity in the general population.

Keywords: Obesity; Overweight; Psychogenic non-epileptic seizures; AED

\section{Introduction}

The incidence of being overweight or obese is more than $60 \%$ in the US population $[1,2]$. The economic burden of physical inactivity worldwide due to being overweight is 67.5 billion dollars [3]. Epilepsy patients have greater risk for obesity due to multiple factors such as metabolic syndrome, the effect of antiepileptic drugs (AEDs) on weight gain, and less physical

Manuscript submitted April 6, 2017, accepted May 2, 2017

${ }^{a}$ Geisinger Healthcare System, Geisinger Commonwealth Medical School, Neuroscience Institute, 100 Academy Ave., Danville, PA 17822, USA

${ }^{b}$ Communication Science Department, Montclair State University, Bloomfield, NJ, USA

${ }^{\mathrm{c} C o r r e s p o n d i n g ~ A u t h o r: ~ A b u h u z i e f a ~ A b u b a k r, ~ G e i s i n g e r ~ H e a l t h c a r e ~ S y s t e m, ~}$ Geisinger Commonwealth Medical School, Neuroscience Institute, 100 Academy Ave., Danville, PA 17822, USA. Email: huzy4tongor@gmail.com

doi: https://doi.org/10.14740/jnr436w activity due to psychosocial issues [4-6]. Further studies demonstrated that obesity is more common in patients with refractory epilepsy and those on polytherapy [7]. Furthermore, brain regions involved in eating behavior are among the most epileptogenic areas, leading to the possibility that people with epilepsy may have altered eating behaviors that contribute to the increased rate of obesity in the epilepsy population [8]. Even though these factors are less likely to affect psychogenic non-epileptic seizure (PNES) patients, there are no reports in the literature about the incidence of obesity in patients with PNES. The difference between PNES and epilepsy patients is no ictal activity in the brain of PNES patients; however, other characteristics between PNES and epilepsy patients are somewhat similar such as psychological and affective disorders and usage of AEDs. Hence, if seizure (electrographic activity of the brain) contribution to weight gain is correct, we would expect that PNES patients would have lower body mass index (BMI) values than epilepsy patients. Also we attempted to evaluate the effects of AEDs on weight gain. By evaluating PNES patients, we removed the role of seizures (electrographic activity of the brain) in weight gain. Therefore, we can more specifically look at whether AEDs properties affect weight gain.

\section{Methods}

We retrospectively reviewed all the medical records of patients admitted to the Epilepsy Monitoring Unit at the University of Mississippi Medical Center from January 2013 to December 2015. Patients with the diagnosis of PNES were ascertained. All patients with PNES underwent prolonged video EEG scalp recording and placebo challenge that precipitated the same constellation of symptoms that the subjects experienced as their habitual spells. Patients with subtle clinical manifestations were excluded. Besides demographic characteristics, we obtained body weight in kilograms and height in meters for the calculation of BMI. Overweight was defined as BMI 25 - 29, obese as BMI $30-39$, and morbidly obese as BMI $>40$. Similarly seizure duration, seizure frequency, and number of AEDs were collected.

\section{Statistical analysis}

A logistic regression was conducted with AED property (positive $=$ inducing weight gain; negative $=$ not inducing weight 
Table 1. Patient's Characteristics

\begin{tabular}{|ll}
\hline Gender & \\
\hline Female & 69 \\
Male & 20 \\
Ethnicity & \\
African American & 47 \\
White & 41 \\
Hispanic & 1 \\
BMI & \\
Normal & 27 \\
Overweight & 22 \\
Obese & 32 \\
Very obese & 8 \\
\hline
\end{tabular}

gain) as the dependent measure $(\alpha<0.05)$. Gender, BMI status, seizure duration, and seizure frequency were entered as predictors in the model.

The study was approved by the Ethic Committee and Institutional Review Board of the UMC.

\section{Results}

A total of 89 patients were selected, with mean age of 33.5 years and age range of $16-64$ years (Table 1). There were 20 males and 69 females $(77.5 \%)$; 41 were Caucasian $(46.1 \%)$, 47 were Afro-American (AA) (52.8\%) and one was Hispanic. The duration of symptoms was between 1 month and 40 years. Eighteen patients had their symptoms for $<1$ year, 43 between 1 and 5 years, 15 between 5 and 10 years, and 13 for over 10 years, and four out of these 13 had their symptoms between 30 and 40 years.

Twenty-seven patients had normal BMI (30.3\%), 22 were overweight $(24.7 \%), 32$ were obese $(36 \%)$ and eight were very obese $(9 \%)$. Overall, 62 patients were considered overweight representing $69.6 \%$ (Table 2 ). Significantly more AA females had overweight or obesity compared to Caucasian females (33 vs. 15) which had the higher rate of normal weight (12 vs. $6)$. This is in contrast to the males where the rate is similar between the Caucasian and AA (6 vs. 7). Overall, the rate of overweight/obesity in PNES is similar to the reported rate of overweight/obesity of $55.2-72 \%$ in epilepsy patients [7, 9].

All patients but six were taking between 1 and 5 AEDs. Thirty-nine patients were on two AEDs, 28 on one AED, 10 on three AEDs, five patients on four AEDs and one was taking five AEDs. Two patients had vagal nerve stimulator implanted, erroneously being misdiagnosed as epileptic. There were 24 patients taking depakote, gabapentin or pregabalin which had a positive effect on weight gain. For the purpose of statistical analysis, the dependent measure of AEDs was converted to a categorical variable, with AEDs with positive effect on body weight being one category and those without effect on body weight being the other category (Table 3 ).

A logistic regression analysis was conducted with seizure
Table 2. Body Weight Analysis Between Caucasian and African American

\begin{tabular}{lll}
\hline & African American & White \\
\hline Female & & \\
Normal weight $(\mathrm{n}=20)$ & 6 & 14 \\
Overweight $(\mathrm{n}=19)$ & 12 & 6 \\
Obese $(\mathrm{n}=24)$ & 16 & 8 \\
$\quad$ Very obese $(\mathrm{n}=6)$ & 5 & 1 \\
Male & & \\
Normal weight $(\mathrm{n}=7)$ & 2 & 5 \\
Overweight $(\mathrm{n}=3)$ & 3 & 0 \\
Obese $(\mathrm{n}=8)$ & 2 & 6 \\
Very obese $(\mathrm{n}=2)$ & 1 & 1 \\
\hline
\end{tabular}

duration (months), seizure frequency (per month), gender, and BMI (normal, overweight, obese and very obese) as predictors and AED property (yes - linked with obesity; no - not linked with obesity) as the dependent factor. All factors were entered simultaneously. None of the predictor variables were linked with the dependent variable (AED property). BMI did not predict the AED properties of the medications the patients were taking. Similarly, there was no significant correlation (Pearson correlation) between the duration of symptoms and $\mathrm{BMI}(\mathrm{P}=$ $0.525)$ or PNES frequency and BMI $(\mathrm{P}=0.339)$.

\section{Discussion}

This study demonstrates that being overweight or obese is prevalent in patients with PNES. To our knowledge, this is a first study 1) evaluating BMI characteristics in PNES patients and comparing it to recent values reported in the literature on epilepsy patients; 2) reporting the impact of AED property on the rate of obesity and overweight in patients with PNES using BMI. First, we found that the rate of overweight and obesity in PNES is similar to the rate reported in epilepsy patients. The overall rate of overweight and obesity in the epilepsy patients ranges between $55.2 \%$ and $72 \%$ [4-6], and the corresponding rate in PNES patients found in this study is $69.3 \%$. Even though the literature has conflicting reports, there is general agreement that there is direct correlation between epilepsy and obesity as well as a trend towards higher rate in AA and females. This is similar to the rate in our cohort of PNES patients.

Ladino et al [9] assessed the BMI in a series of patients with idiopathic epilepsy to determine the impact of epilepsy on BMI. They concluded that there was an association between epilepsy and body weight, even though their patients were physically active. Hence they concluded that there was no clear correlation between obesity and exercise but rather that epilepsy was directly related to obesity. Similarly, they found that obesity is directly correlated to family history of epilepsy and there was no difference between drug-resistant among obese and non-obese patients. However, in our PNES 
Table 3. Average Values for BMI, Age, Seizure Duration and Seizure Frequency as a Function of AED Characteristics (Negative or Positive Link With Weight Gain)

\begin{tabular}{lll}
\hline & $\begin{array}{l}\text { Positive AED } \\
(\mathbf{n}=\mathbf{2 4})\end{array}$ & $\begin{array}{l}\text { Negative AED } \\
(\mathbf{n}=65)\end{array}$ \\
\hline BMI & 28.15 & 30.389 \\
Age & 39.833 & 31.185 \\
Seizure duration (months) & 49.83 & 74.29 \\
Seizure frequency per month & 20.25 & 36.26 \\
\hline
\end{tabular}

patients, we found the rate of overweight and obesity is similar to that of those with epilepsy patients $69 \%$ vs. $72 \%$ respectively, despite the absence of iterictal/ictal activity in the brain that may stimulate various networks which change the appetite.

In an attempt to explain the higher rate of obesity in epilepsy patients, Small et al [8] carried out an experiment examining the central processing of taste and concluded that taste sensation is processed at the orbitofrontal cortex as well as anteromedial temporal lobe and implicates the right hemisphere for the higher order function. Overall they suggested that stimulation of these regions by the epileptic electrical activity changes the patient's appetite and contributes to the higher rate of obesity. Again we found similar rate of obesity in PNES patients which contradict this hypothesis.

Interestingly we did not find any correlation between obesity and AEDs that are known to cause weight gain such as valproate or gabapentin. Similarly there were no correlations between duration of or the frequency of the events and weight. These findings were similar to the report by Janousek et al [7] in their epilepsy population. Again this may suggest that obesity is part of the general trend of weight gain in the society rather epilepsy per se.

In conclusion, our findings suggest that being overweight/ obese may be part of general trends in the society and less likely that it is related to epilepsy as has been reported in many series. Of course the short coming of our study is that it is retrospective. Also, we did not assess many variables such as physical activity or caloric intake. However, we conclude that the rate of obesity is similar in PNES and epilepsy patients.

\section{References}

1. Flegal KM, Carroll MD, Ogden CL, Curtin LR. Prevalence and trends in obesity among US adults, 1999-2008. JAMA. 2010;303(3):235-241.

2. Flegal KM, Carroll MD, Kit BK, Ogden CL. Prevalence of obesity and trends in the distribution of body mass index among US adults, 1999-2010. JAMA. 2012;307(5):491497.

3. Ding D, Lawson KD, Kolbe-Alexander TL, Finkelstein EA, Katzmarzyk PT, van Mechelen W, Pratt M, et al. The economic burden of physical inactivity: a global analysis of major non-communicable diseases. Lancet. 2016;388(10051):1311-1324.

4. Kobau R, Zahran H, Grant D, Thurman DJ, Price PH, Zack MM. Prevalence of active epilepsy and health-related quality of life among adults with self-reported epilepsy in California: California Health Interview Survey, 2003. Epilepsia. 2007;48(10):1904-1913.

5. Kobau R, Zahran H, Thurman DJ, Zack MM, Henry TR, Schachter SC, Price PH, et al. Epilepsy surveillance among adults - 19 States, Behavioral Risk Factor Surveillance System, 2005. MMWR Surveill Summ. 2008;57(6):1-20.

6. Nair SS, Harikrishnan S, Sarma SP, Thomas SV. Metabolic syndrome in young adult with epilepsy. Seizure. 2016;37:61-64.

7. Janousek J, Barber A, Goldman L, Klein P. Obesity in adults with epilepsy. Epilepsy Behav. 2013;28(3):391394.

8. Small DM, Jones-Gotman M, Zatorre RJ, Petrides M, Evans AC. A role for the right anterior temporal lobe in taste quality recognition. J Neurosci. 1997;17(13):5136-5142.

9. Ladino LD, Hernandez-Ronquillo L, Tellez-Zenteno JF. Obesity and its association with generalised epilepsy, idiopathic syndrome, and family history of epilepsy. Epileptic Disord. 2014;16(3):343-353. 